\title{
Comparative evaluation of the results of treatment of acute adhesive bowel obstruction in children
}

\begin{abstract}
100 endosurgical and 100 traditional surgery methods were performed, it was established that a comparative analysis of laparoscopic and traditional methods of treatment showed that endoscopic adhesion has a significant advantage over traditional laparotomy.

Keywords: endosurgical, laparoscopic, adhesion, adhesions, relapratamia, tramotism
\end{abstract}

\author{
Volume 10 Issue I - 2018
}

\author{
Kobilov Ergash Egamberdievich \\ Samarkand State University, Samarkand, Republic of Uzbekistan \\ Correspondence: Kobilov Ergash Egamberdievich, Samarkand \\ State University, Samarkand, Republic of Uzbekistan, \\ Email kobilov.196।@mail.com
}

Received: November 08, 2017 | Published: January 09, 2018

\section{Introduction}

Treatment of acute adhesive bowel obstruction (AABO) in children is a complex task, as the causes and reports of the disease are extremely diverse. It should be noted that the condition of the abdominal cavity in patients with AABO is not always subordinated to any particular stereotype, so therapeutic tactics are selected individually, taking into account the severity of the disease and the state of the abdominal cavity. We consider it fundamentally important that for the development of treatment tactics, such factors as the severity of the disease, the severity of the intestinal paresis, the prevalence of the adhesion process and the frequency of relapses should play an important role. In the last decade in pediatric surgery, and in recent years and in general surgery, work has appeared in which laparoscopy has been successfully used for the diagnosis and treatment of $\mathrm{AABO}$. However, these studies are few in number, and they evaluate the possibilities of the laparoscopic method in different ways. Most surgeons believe that the use of laparoscopy in these cases is not only impractical, but also dangerous because of the possibility of iatrogenic damage to the intestine in conditions of paresis and adhesions in the abdominal cavity.

\section{Materials and methods of research}

To evaluate the effectiveness of the endoscopic method of diagnosis and treatment of $\mathrm{AABO}$, we compared the results of treatment in two groups of patients: the main (endosurgical) and the comparison group ("open" - traditional). The main group included 100 patients who underwent endosurgical interventions. The control (comparative) group was also made by 100 patients who underwent "open" (traditional) surgical operations. Selection of 100 patients in both groups was performed arbitrarily.

\section{Results and discussion}

When assessing the effectiveness of laparoscopy, we compared the nearest postoperative period in these groups of patients. For the characterization of the immediate postoperative period, the following criteria were used: the patient's general well-being and physical condition, the time of occurrence of active peristalsis determined by auscultation, the volume of stagnant contents in the stomach and the dynamics of its decrease, the time of appearance of the stool, and the number of days spent by the patient in the hospital after operative treatment. Laparoscopic adhesion in comparison with the traditional surgical intervention was characterized by minimal intraoperative blood loss, a short duration of operations: $41.6 \pm 13.4$ min versus 85.8 \pm 11.7 min with the traditional method of treatment. A careful analysis of our clinical observations has convincingly proved that the low traumatism of laparoscopic operations in comparison with traditional surgical interventions has a positive effect on the overall health and well-being of children. Minimal injury of the anterior abdominal wall and abdominal organs contributes to a more smooth course of the postoperative period. The pain syndrome in all patients was not expressed and was easily stopped by a single or double injection of analgesics. After laparoscopic operations, the pain syndrome persisted to the end of the first day in 92 patients $(92 \%)$, on the second day - in 51 patients $(51 \%)$, on the third - in 13 children $(13 \%)$ and almost completely stopped on the fourth day. In patients operated in the traditional way (control group), the pain syndrome persisted for a longer period: in all children it was observed in the first two days, on the third day in 82 children (82\%), on days $4-5$ in 66 children $66 \%$ ), and in some patients the pain syndrome persisted even on the $5^{\text {th }}-7^{\text {th }}$ day.

Decline of the pain syndrome contributed to the rapid recovery of physical activity of patients - patients started walking 1-4 days after laparoscopic surgery (on average - after 2.1 days). The motor activity of patients in the control group was significantly reduced. On the second day, only 6 patients $(6 \%)$ started to walk, and 59 patients $(59 \%)$, the third -18 children $(18 \%)$, the remaining patients on the $5^{\text {th }}-7^{\text {th }}$ day after surgery.

The most distinct advantage of the laparoscopic technique was the rapid restoration of normal peristalsis of the intestines, a much less pronounced postoperative paresis. It should be noted that in none of our cases there has been an intubation of the small intestine. When comparing the results obtained, it was found that in the main group, the stagnant contents of the stomach in a volume of $24.3 \pm 1.6 \mathrm{ml}$ were revealed in the first hours after the operation in 14 patients (14\%). With subsequent probing, stagnant contents were not detected, which allowed the initiation of early enteral feeding. In the control group, stagnant contents in the stomach in the first day were found in $100 \%$ of patients, in the second group - in 66 patients $(66 \%)$, on the third in 13 children (13\%). The disappearance of stagnant contents in the stomach correlated with the timing of intestinal peristalsis. According to our data, the recovery of peristalsis in the main group occurred 2-3days earlier than in the control group.

Appearance of stool in the main group was noted at a time $2.2 \pm$ 0.6 days after the operation, in the control group $-4.1 \pm 0.5$ days. In connection with a more smooth course of the postoperative period, 
the duration of the stay of patients in the intensive care unit $(1.7 \pm$ 0.8 days) in the main group decreased, while in the control group the time spent in the intensive care unit was $2.9 \pm 0.9$ days.

Significantly reduced the time of hospitalization after surgery in the main group ( $6.9 \pm 1.4$ days $)$ compared with the control ( $16.2 \pm 1.9$ days $)$. Using the possibilities of laparoscopic surgery allowed to minimize the possibility of occurrence of postoperative complications, greatly facilitating the course of the postoperative period (Table 1). ${ }^{1-5}$

As can be seen from the table, the total number of early postoperative intra-abdominal complications in the control group was more than 7 times higher than in the main group $(22 \%$ and $3 \%$, respectively). It is worth to pay special attention to a high percentage of such severe complications as relaparotomy and intestinal event. Relaparotomy was performed with the relapse of the early acute adhesive bowel obstruction ( 4 observations) and in connection with the development of purulent peritonitis due to insufficiency of gastrostomy, which was imposed for intubation of the small intestine (one observation). It should also be pointed out that a high level of inflammatory complications from the postoperative wound and abdominal cavity (13\%). In the main group, the overall level of postoperative complications was only $3 \%$. In one patient in the immediate postoperative period after laparoscopic separation of adhesions, progression of obstruction was noted, which was associated with an incorrect determination during the intervention of the site of obstruction. Laparotomy was performed, separation of adhesions, the child recovered. Suppuration of the puncture site of the anterior abdominal wall in the umbilical region was noted in one patient. Ligature fistula also appeared in one observation.

Table I Postoperative complications with PSC in the main and control groups

\begin{tabular}{llll}
\hline \multirow{2}{*}{ Complications } & Patient groups & Total \\
\cline { 2 - 4 } & Main $\mathbf{n = 1 0 0}$ & Control $\mathbf{n = 1 0 0}$ & \\
\hline Relaparotomy & $\mathrm{I}$ & 5 & 6 \\
Eventration & 0 & 4 & 4 \\
Abscess of small pelvis & 0 & 2 & 2 \\
Abdominal infiltrate & 0 & $\mathrm{I}$ & $\mathrm{I}$ \\
Suppuration of a postoperative wound & $\mathrm{I}$ & 6 & 7 \\
Ligature fistulas & $\mathrm{I}$ & 4 & 5 \\
Total & 3 & 22 & 25 \\
\hline
\end{tabular}

\section{Conclusion}

The use of laparoscopic technologies in the complex treatment of patients with acute obstructive pulmonary disease has made it possible to reduce the costs of intensive care in the intensive care and surgical departments and has given a certain economic effect in comparison with the traditional method of treatment, primarily by improving the quality of surgical intervention and reducing the volume of intensive therapy, terms of postoperative treatment due to the low traumatic nature of the operation. Comparative analysis of laparoscopic and traditional methods of treatment showed that endoscopic adhesion has significant advantages over traditional laparotomy.

\section{Acknowledgments}

None.

\section{Conflicts of interest}

None.

\section{References}

1. Blinnikov OI, Dronov AF, Smirnov AN. Laparoscopic surgery for acute adhesive intestinal obstruction in children. Laparoscopic surgery (Laparoskopik khirurgiya). 1993;82-88.

2. Dronov AF, Poddubny IV, Blinnikov OI. Laparoscopic surgery for acute adhesive intestinal obstruction in children. Surgery (Khirurgiya). 2001;2:37-42.

3. Isakov Yu F, Stepanov EA, Dronov AF. Laparoscopy in the treatment of acute adhesive intestinal obstruction in children. Surgery (Khirurgiya). 1985;7:11-14.

4. Kotlobovsky VI, Dronov AF, Poddubny IV, et al. Comparative evaluation of the results of treatment of common forms of appendicular peritonitis in children, operated by laparoscopic and traditional methods. Surgery (Khirurgiya). 2003;N7: 32-37.

5. Rekhachev VP, Nedashkovsky EV. To the ratio of conservative and operative approaches in the treatment of adhesive acute intestinal obstruction. Intestinal obstruction, Novosibirsk. 1993;92-94. 